\title{
Prevention of Intradialytic Hypotension with Intermittent Back-Filtrate Infusion Haemodiafiltration: Insights into the Underlying Mechanism
}

\author{
Yutaka Koda ${ }^{a}$ Ikuo Aoike ${ }^{b}$ \\ ${ }^{a}$ Koda Medical and Dialysis Clinic, Tsubame, Japan; ${ }^{b}$ Koyo Medical Clinic, Kamedakoyo, Japan
}

\section{Keywords}

Intradialytic hypotension - Intermittent infusion .

Sympathetic activation · Plasma refilling

\begin{abstract}
Background: Intradialytic hypotension (IDH) is a major challenge to safely performing haemodialysis. Blood volume depletion due to fluid removal is a major cause of hypotension, so more emphasis should be placed on finding alternative modalities to traditional constant rate ultrafiltration. Summary: Intermittent back-filtrate infusion haemodiafiltration (I-HDF) utilises purified online quality dialysate with an automated dialysis machine. A bolus of $200 \mathrm{~mL}$ of dialysate is repetitively infused at 30-min intervals. A pilot study with 68 hypotension-prone patients revealed that I-HDF can reduce the frequency of IDH interventions, particularly in elderly patients and patients with large interdialytic weight gain (IDWG). This was typically accompanied by an increase in intradialytic blood pressure and decreased tachycardia in the latter half of the session, suggesting reduced sympathetic stimulation during I-HDF. Protective mechanisms involved in the pathophysiology of IDH could be explained in part by the findings obtained in this pilot study. Intermittent increases in blood pressure during I-HDF may prevent venous
\end{abstract}

pooling (i.e., the DeJager-Krogh phenomenon), and reduced sympathetic stimulation may maintain a physiological state less likely to induce the cardio-vagal reflex (i.e., the BezoldJarisch reflex). The plasma refilling rate (PRR), evaluated as the refilling fraction (RF), is unexpectedly smaller in I-HDF. However, in patients who respond, the RF is well achieved, which suggests that adequate PRR is the central physiology for preventing IDH. Patients for whom I-HDF is effective are characteristically relatively elderly and show increased IDWG. Blood pressure increment and reduced sympathetic activation in I-HDF may be a mechanism for prevention of IDH. Key Messages: Evaluating relative changes in blood volume during I-HDF will provide a new perspective for exploring appropriate ultrafiltration modification that circumvents IDH.

(c) 2019 S. Karger AG, Basel

\section{Introduction}

Intradialytic hypotension (IDH) is a commonly encountered challenge in the field of dialysis medicine and remains a clinical feature that is difficult to manage. Haemodiafiltration (HDF) has been predicted to be a preventive method for dialysis-induced hypotension. Pre-dilu-

\section{KARGER}

(c) 2019 S. Karger AG, Basel

E-Mail karger@karger.com

www.karger.com/bpu
Yutaka Koda

Koda Medical and Dialysis Clinic

3748 Yoshida

Tsubame, Niigata 959-0264 (Japan)

E-Mail ykodan@gmail.com 
tion on-line HDF was once reported to be effective but is debatable as a first-line option [1].

The current definitions of HDF are multifaceted and unstandardised. In Japan, HDF is generally accepted as intentional filtration of $>5 \mathrm{~L}$ excluding interdialytic weight gain (IDWG). In Europe, it is proposed that HDF involves ultrafiltration of $>20 \%$ of the blood volume processed [2]. The volume of ultrafiltration during intermittent infusion HDF (I-HDF) satisfies neither of these definitions, so whether these definitions can be uniformly applied to HDF is a matter of debate. However, because I-HDF is a treatment modality in which infusion and ultrafiltration are performed in a closed system, Japanese medical insurance accepts this procedure as HDF if equipment requirements are met. In I-HDF mode, accumulated body fluid is removed while simultaneously increasing vascular volume via intermittent replenishment with bolus back-filtrate infusion. Pulsed profile ultrafiltration is not recommended by the European Best Practice Guidelines [3], but I-HDF differs in that it requires repetitive infusion. A preventive effect against IDH was previously reported in I-HDF mode [4].

\section{Importance of Countermeasures against IDH}

For dialysis patients, accumulated body fluid (typically $3-5 \%$ of body weight) must be removed during each dialysis treatment, usually performed thrice weekly; thus, blood volume is increased and decreased repeatedly. This non-physiological fluctuation may cause stressful blood pressure instability. IDH is a phenomenon that occurs when the cardiovascular response cannot compensate for hypovolemia caused by excessive or rapid fluid removal.

The definition of IDH remains inconsistent and differs within the KDOQI, European Best Practice Guidelines, and HEMO study groups [5]. The definition of IDH by the KDOQI involves a drop in systolic blood pressure (SBP) by $20 \mathrm{~mm} \mathrm{Hg}$ or more or a drop in mean blood pressure by $10 \mathrm{~mm} \mathrm{Hg}$ associated with various symptoms of hypotension [6].

In addition to the decline in QOL, coronary and cerebral blood flow rates rapidly decrease during IDH; therefore, IDH is recognised as a mortality risk. In terms of mortality, the lowest SBP point (i.e., nadir) should not fall below $90 \mathrm{~mm} \mathrm{Hg}$ [5] or should not decrease by $>40 \mathrm{~mm} \mathrm{Hg}$ [7]. At present, IDH prevention is still one of the major challenges in intermittent renal replacement therapy.

The main causes of IDH attributable to patient factors can be divided into 3 categories. First, blood volume-relat- ed factors are substantial contributors to hypotension and include ultrafiltration volume, ultrafiltration rate (UFR), and plasma refilling rate (PRR). Second, vascular tone is a clear cause and includes sympathetic dysfunction, body temperature, and blood redistribution due to meals during dialysis. The third main cause is cardiac factors, such as ischaemic heart disease, diastolic dysfunction, arrhythmia, myocardial stunning, and regional wall motion abnormalities. Among these, a large ultrafiltration volume is likely to be the primary contributing factor in the induction of IDH.

Conventionally, it has been shown that patients who develop IDH recover with rapid replenishment after the administration of 100-200 mL of normal saline. Indeed, some long-term experienced dialysis patients sometimes request a bolus saline infusion during the latter half of dialysis in order to prevent morbidity, despite a lack of significant drop in blood pressure. These common occurrences have provided a background for efforts to advance I-HDF therapy [8].

\section{Application of I-HDF for Hypotension-Prone Patients}

In the default mode of I-HDF, $200 \mathrm{~mL}$ of dialysate is rapidly infused by backward filtration through a diafilter membrane at a rate of $150 \mathrm{~mL} / \mathrm{min}$ at 30 -min intervals using a programmable system for an automated dialysis machine [4]. In other words, ultrafiltration with simultaneous infusion is performed with fine-tuned graded increases in the time-averaged blood volume, which theoretically may be advantageous for maintaining blood pressure if balanced with PRR. Thus, I-HDF is a volume-centred approach to counter cardiovascular instability as well as an anti-fouling approach to diafiltration membranes by virtue of the repetitive backwash that occurs during convective therapy $[4,8]$.

Both elderly patients and those with large weight gain would particularly benefit from I-HDF treatment [4]. In our previous multicentre prospective pilot study that used a crossover method for hypotension-prone patients, the frequency of interventions was reduced by $18 \%$ [4]. Multivariate analysis in the study demonstrated that I-HDF was beneficial in elderly people (aged $>70$ years) and in patients with high IDWG (i.e., $\geq 4 \%$ of dry body weight). In univariate analysis, patients with longer session times and those taking pressor agents were found likely to respond to I-HDF. The most important findings of the study were that SBP at $5 \mathrm{~min}$ after infusion was high with an average of $4 \mathrm{~mm} \mathrm{Hg}$ during I-HDF and that the pulse rate increment during the latter half of the session was decreased with I-HDF, in comparison with conven- 


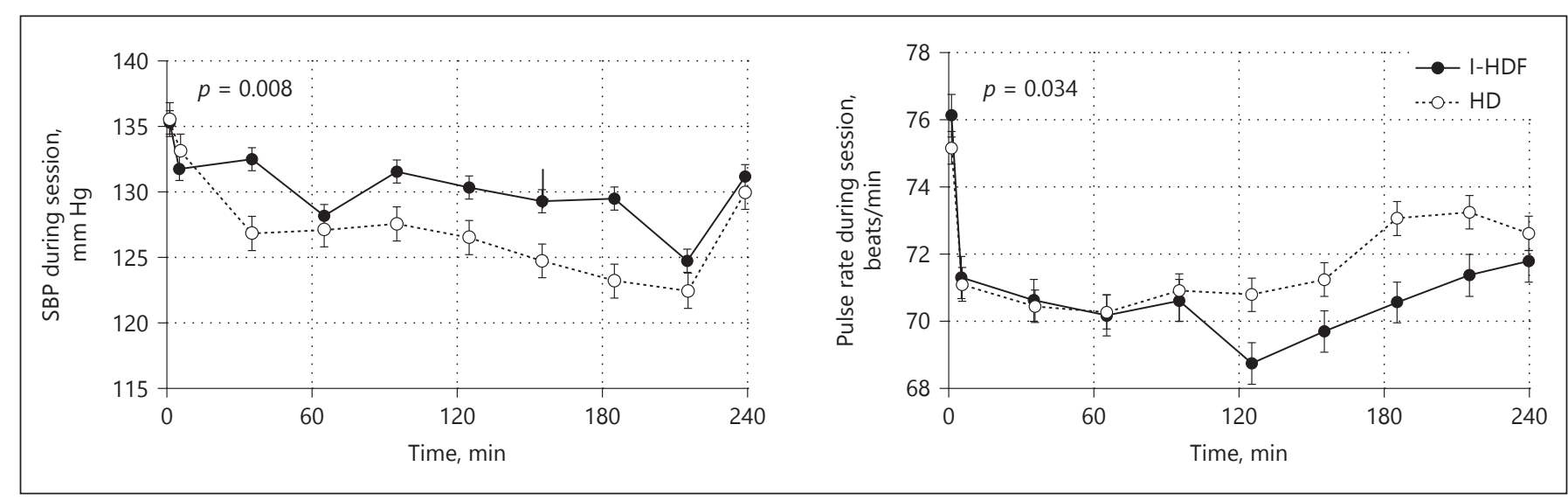

Fig. 1. SBP and pulse rate changes during HD and I-HDF sessions. Pulse rate was significantly lower during the latter half of I-HDF, suggesting lower sympathetic stimulation [4]. SBP, systolic blood pressure; I-HDF, infusion haemodiafiltration.

tional dialysis. This indicates a gradual increase in sympathetic tone accompanied by ultrafiltration, which is considerably less than that observed in dialysis with constant-rate ultrafiltration (Fig. 1). These findings may explain the mechanisms underlying how I-HDF prevents IDH, as explained in the next section.

\section{Pathophysiological Role of I-HDF in Prevention of IDH}

\section{Prevention of Venous Pooling}

Reduction of blood flow in muscle, skin, and especially the visceral organs induces constriction in the arterial side of the capillary bed, which plays a role in peripheral resistance, in order to maintain blood pressure. However, if the maximal limit in the visceral organs is reached, ischaemia may occur with adenosine release, causing significant reduction in small vasculature tone [9]. This reduction in arteriolar tone leads to an increase in downstream distending pressure resulting in local venular capillary expansion. The venular blood pooling diminishes venous return to the heart, consequently reducing cardiac output and blood pressure (i.e., the DeJager-Krogh phenomenon) [10]. The post-infusion rise in SBP during I-HDF might prevent this phenomenon by circumventing organ ischaemia and adenosine release.

\section{Prevention of Cardiac Inhibitory Vagal Reflex}

In general, tachycardia in response to ultrafiltration is undesirable because it shortens diastole, reducing left ventricular filling and coronary perfusion, both of which occur only during diastole. With progressive hypovolemia induced by ultrafiltration, blood volume and cardiac filling become critically reduced and compensatory sympathetic activation ensues, seen as tachycardia and vasoconstriction. Then the activation of myocardial mechanoreceptors, which are stimulated by vigorous contractions of the underfilled left ventricle, eventually induces sympathetic inhibition to protect the heart muscle itself, resulting in sudden vasodilation, bradycardia, and severe hypotension (i.e., the Bezold-Jarisch reflex) [11]. This reflex is typically preceded by extreme sympathoactivation. Thus, decreased sympathetic activation during the latter half of I-HDF might play an important role in preventing IDH [4].

\section{Does PRR Increase in I-HDF?}

PRR represents the transfer of fluid from the interstitium into the vascular lumen. According to Starling's classic hypothesis of transvascular fluid dynamics, this transfer is caused by the balance of hydrostatic pressure and colloid osmotic pressure in the capillary bed. Strictly speaking, the confluence oflymphatic flow into the venous angle is also included in plasma refilling. According to estimations using Guytonian pressure-volume curves of interstitial space, an increase in the extracellular fluid volume of about 5\% (corresponding to typical IDWG in dialysis patients) linearly increases interstitial pressure [12]. In other words, the greater the increase in body weight during the non-dialysis period, the greater the increase in interstitial pressure, resulting in higher PR. Although it has been proposed that the filtration coefficient of the cap- 
Fig. 2. Estimation of plasma refilling $\left(Q_{p r}\right)$ based on hematocrit change before and after dialysis, and reference blood volume [13]. Q pr: PRR (mL/min), Quf: UFR (mL/ $\min$ ), $\mathrm{Ht}_{0}$ : $\mathrm{Ht}$ at dialysis start, $\mathrm{Ht}_{\mathrm{t}}$ : $\mathrm{Ht}$ after $t$ minutes, $\mathrm{V}_{\mathrm{RBC}}$ : $\mathrm{RBC}$ volume, $\mathrm{Vp}$ : plasma volume $(\mathrm{mL}), \mathrm{Vp}_{0}$ : plasma volume at dialysis start $(\mathrm{mL}), \mathrm{Vp}_{\mathrm{t}}$ : plasma volume after $t$ minutes $(\mathrm{mL}) . \Delta \mathrm{Vp}$ : plasma volume change $(\mathrm{mL}), \mathrm{TBV}_{\mathrm{t}}$ : total blood volume after $t$ minutes $(\mathrm{mL})$. Ht, haematocrit.

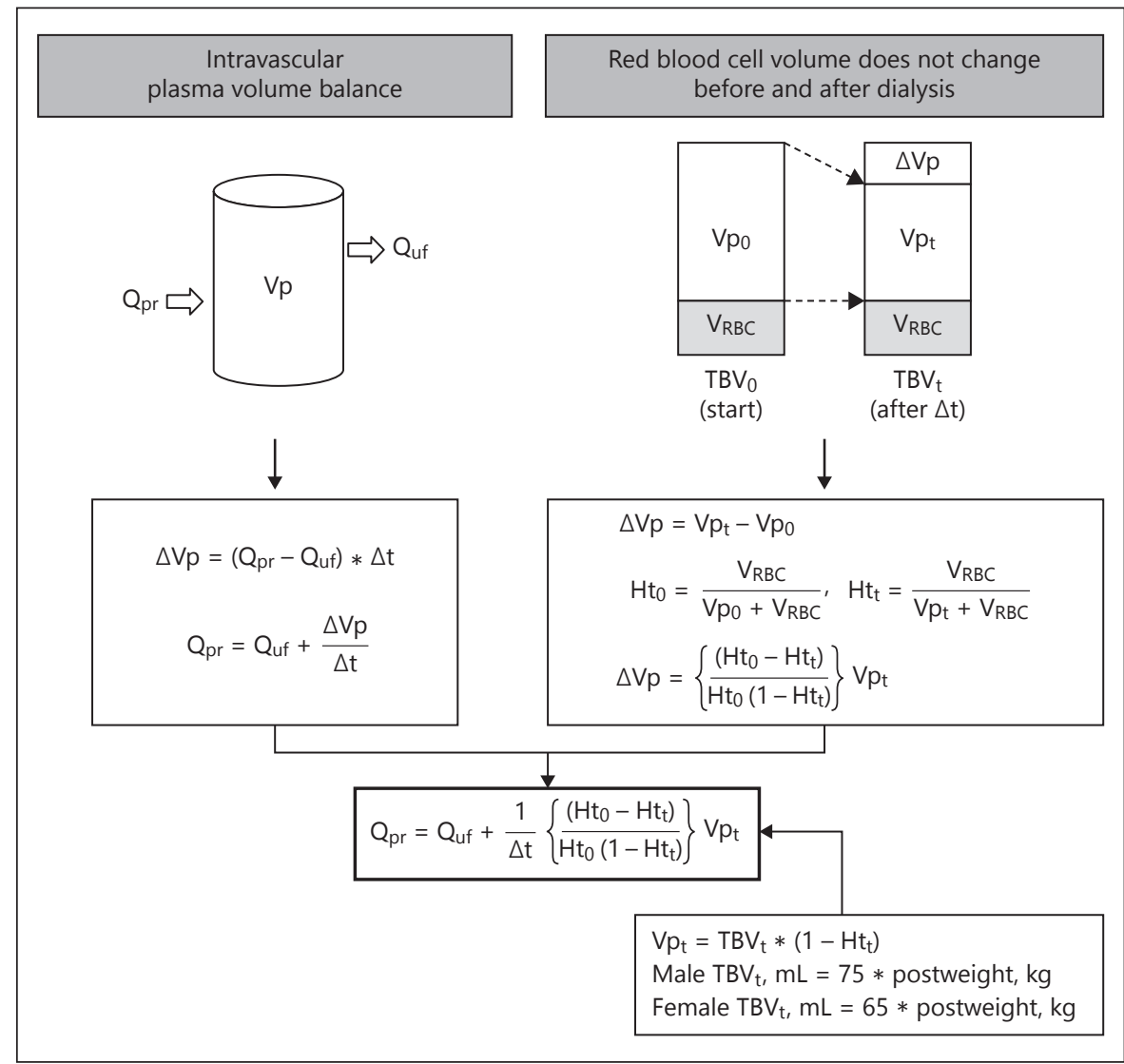

illary wall is constant if there is no inflammation, the possibility of improving PRR is promising because the repetitive infusion during I-HDF causes an increase in the capillary surface area. However, there is currently insufficient data and the hypothesis remains a subject of debate.

\section{Estimation of PRR}

As a secondary analysis of our multicentre prospective pilot study [4], we examined PRR based on haematocrit (Ht) values before and after dialysis. PRR was calculated based on the premise that the volume of erythrocytes is unchanged and that body fluid volume returns to within normal range and is evenly distributed after dialysis, and thus a normal absolute blood volume is adopted [13]. The Fåhræus effect was not taken into consideration. Clinical evaluation of PRR was performed in terms of the refilling fraction (RF; PRR/UFR, Qpr/Quf; Fig. 2).

$\mathrm{RF}$ was significantly higher in $\mathrm{HD}$, contrary to expectations (HD $0.79 \pm 0.04$, I-HDF $0.74 \pm 0.03, p<0.05$, average $\pm \mathrm{SD}, n=64)$. However, when comparing between the changes that were seen in the success and failure groups, the RF was increased during the I-HDF phase in the success group but was decreased in the failure group (Fig. 3). This indicates that the I-HDF effect of reducing the frequency of interventions is significantly associated with RF, and the probability of success is unreliable when $\mathrm{RF}$ is small. In addition, since the IDWG of the success group was large, it is conceivable that interstitial fluid retention has a strong influence on PRR.

\section{Estimation of Standardised Blood Volume by Bolus Infusion}

I-HDF involves repeated bolus infusion several times per session, and each relative blood volume (RBV) change obtained instantaneously at the time of infusion could provide useful information. If standardised blood volume (stdBV) can be maintained at $>65 \mathrm{~mL} / \mathrm{kg}$, there will be few morbid events during dialysis [14]. The stdBV ( $\mathrm{mL} / \mathrm{kg})$ can be monitored at each infusion, 7 times per session, and is estimated using the following theoretical equation:

$$
\operatorname{stdBV}=(\text { bolus infusion }) /\left(\left[\mathrm{Ht}_{0} / \mathrm{Ht}_{\mathrm{t}}-1\right] \text { * body weight }\right) \text {, }
$$



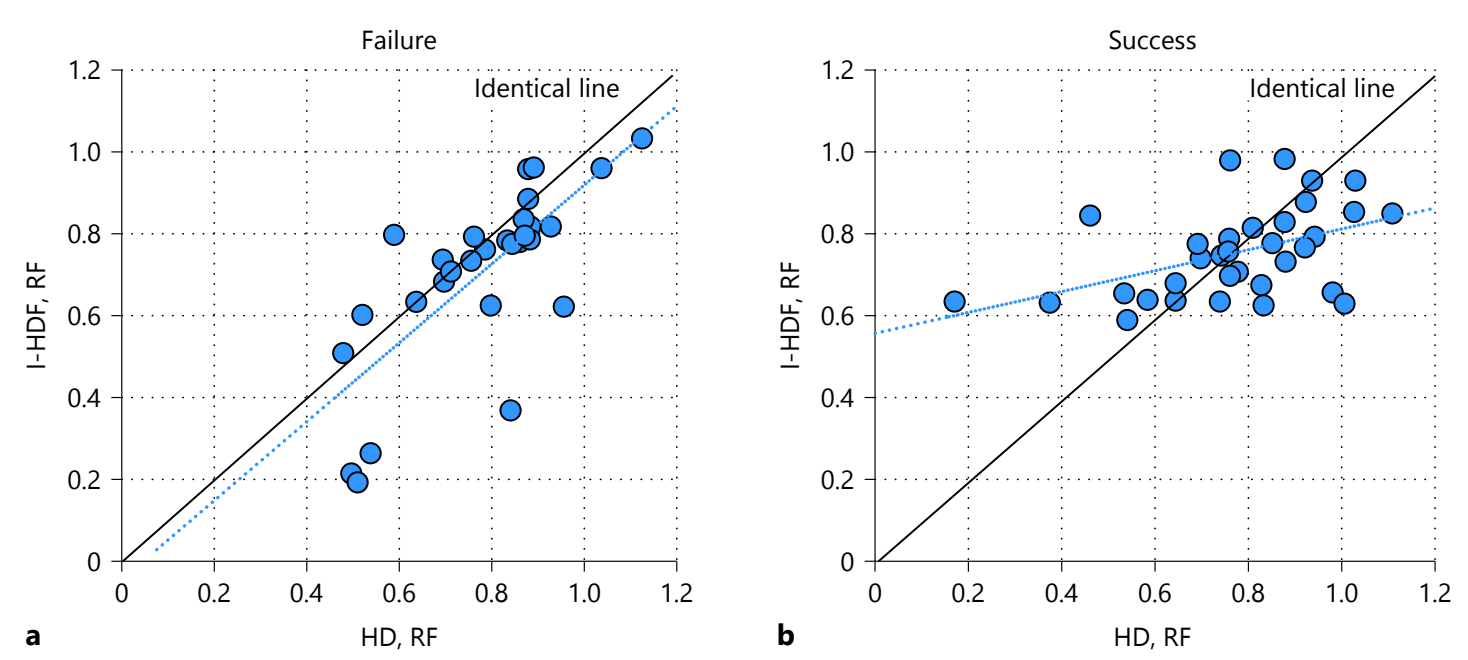

Fig. 3. Comparison of $R F\left(Q_{p r} / Q_{u f}\right)$ between success and failure cases when $H D$ and I-HDF were performed in a crossover manner. RF in the success group (b) was well maintained during I-HDF, with an RF value $>0.6$; this was not observed in the failure group (a). I-HDF, infusion haemodiafiltration; RF, refilling fraction.

where $\mathrm{Ht}_{0}$ and $\mathrm{Ht}_{\mathrm{t}}$ are $\mathrm{Ht}$ before and after bolus infusion.

Moreover, the presence of markedly high dilution occurs after bolus infusion would suggest recirculating blood flow. Thus, I-HDF with frequent RBV monitoring provides useful information, including PRR, absolute blood volume estimation, and recirculation detection.

\section{Conclusion}

In some cases of hypotension, the frequency of interventions for IDH was reduced with the introduction of I-HDF. The characteristics of patients who responded to I-HDF included relatively old age and increased IDWG above average. Reduced sympathetic activation may be a mechanism in the prevention of IDH, especially via the Bezold-Jarisch reflex. Evaluating RBV changes during I-HDF will provide a new perspective for exploring adequate water removal programmes. Future studies on I-HDF will involve elucidating methods to proceed with a personalised programmed UFR that offers appropriate fluid removal during dialysis.

\section{Acknowledgement}

None.

\section{Statement of Ethics}

Ethics review board approval was not required for this review article.

\section{Disclosure Statement}

The authors have no conflicts of interest to declare.

\section{Funding Sources}

This research received no specific grant from any funding agency in the public, commercial, or not-for-profit sectors.

\section{Author Contributions}

Y.K. conceived of the study and wrote the manuscript. I.A. contributed to data collection and statistical analysis.

References
1 Locatelli F, Altieri P, Andrulli S, Bolasco P, Sau G, Pedrini LA, et al. Hemofiltration and hemodiafiltration reduce intradialytic hypotension in ESRD. J Am Soc Nephrol. 2010 Oct;21(10):1798-807.

2 Tattersall JE, Ward RA, Canaud B, Blankestijn PJ, Bots M, Covic A, et al.; EUDIAL group. Online haemodiafiltration: definition, dose quantification and safety revisited. Nephrol Dial Transplant. 2013 Mar;28(3):542-50. 
3 Kooman J, Basci A, Pizzarelli F, Canaud B, Haage P, Fouque D, et al. EBPG guideline on haemodynamic instability. Nephrol Dial Transplant. 2007 May;22 suppl 2:ii2244.

4 Koda Y, Aoike I, Hasegawa S, Osawa Y, Nakagawa $Y$, Iwabuchi F, et al. Feasibility of intermittent back-filtrate infusion hemodiafiltration to reduce intradialytic hypotension in patients with cardiovascular instability: a pilot study. Clin Exp Nephrol. 2017 Apr;21(2): 324-32.

5 Flythe JE, Xue H, Lynch KE, Curhan GC, Brunelli SM. Association of mortality risk with various definitions of intradialytic hypotension. J Am Soc Nephrol. 2015 Mar;26(3): 724-34.
$6 \mathrm{~K} / \mathrm{DOQI}$ Workgroup. K/DOQI clinical practice guidelines for cardiovascular disease in dialysis patients. Am J Kidney Dis. 2005 Apr; 45(4 suppl 3):S1-153.

7 Shoji T, Tsubakihara Y, Fujii M, Imai E. Hemodialysis-associated hypotension as an independent risk factor for two-year mortality in hemodialysis patients. Kidney Int. 2004 Sep;66(3):1212-20.

8 Mineshima M, Eguchi K. Development of intermittent infusion hemodiafiltration using ultrapure dialysis fluid with an automated dialysis machine. Blood Purif. 2013;35(suppl 1): 55-8.

9 Shinzato T, Miwa M, Nakai S, Morita H, Odani $\mathrm{H}$, Inoue I, et al. Role of adenosine in dialysis-induced hypotension. J Am Soc Nephrol. 1994 Jun;4(12):1987-94.

10 Daugirdas JT. Dialysis hypotension: a hemodynamic analysis. Kidney Int. 1991 Feb;39(2): $233-46$.
11 Barnas MG, Boer WH, Koomans HA. Hemodynamic patterns and spectral analysis of heart rate variability during dialysis hypotension. J Am Soc Nephrol. 1999 Dec;10(12): 2577-84.

12 Guyton AC. Interstitial pressure. II. Pressurevolume curves of interstitial space. Circ Res. 1965 May;16(5):452-60.

13 Butterworth JF, Mackey DC, Wasnick JD. Morgan \& Mikhail's clinical anesthesiology. 5th ed. New York: McGraw-Hill Education; 2013. p. 1168.

14 Kron S, Schneditz D, Czerny J, Leimbach T, Budde K, Kron J. Adjustment of target weight based on absolute blood volume reduces the frequency of intradialytic morbid events. Hemodial Int. 2018 Apr;22(2):254-60. 